\title{
LE DÉSORDRE, ESSENCE OU TOMBEAU DE LA DÉMOCRATIE ? PHILOSOPHER AU PRIX DU DOUTE
}

\author{
Manuel Cervera-Marzal
}

Presses Universitaires de France | « Cités »

2020/3 $N^{\circ} 83$ | pages 13 à 23

ISSN 1299-5495

ISBN 9782130823056

Article disponible en ligne à l'adresse :

https://www.cairn.info/revue-cites-2020-3-page-13.htm

Distribution électronique Cairn.info pour Presses Universitaires de France.

(C) Presses Universitaires de France. Tous droits réservés pour tous pays.

La reproduction ou représentation de cet article, notamment par photocopie, n'est autorisée que dans les limites des conditions générales d'utilisation du site ou, le cas échéant, des conditions générales de la licence souscrite par votre établissement. Toute autre reproduction ou représentation, en tout ou partie, sous quelque forme et de quelque manière que ce soit, est interdite sauf accord préalable et écrit de l'éditeur, en dehors des cas prévus par la législation en vigueur en France. Il est précisé que son stockage dans une base de données est également interdit. 


\section{Le désordre, essence ou tombeau de la démocratie? Philosopher au prix du doute}

MANUEL CERVERA-MARZAL

Pour Leo Strauss, l'un des meilleurs historiens de la discipline, « la philosophie politique est la tentative de connaitre vraiment à la fois la nature des choses politiques et l'ordre politique juste ou bon ${ }^{1}$ ". En effet, de Platon à John Rawls en passant par Thomas Hobbes, la tradition philosophique a pensé la politique du côté de l'ordre, entendu au double sens du terme : commandement ("faites ce que je vous dis") et ordonnancement (" chacun à sa place »). Le désordre - qu'il se manifeste sous la forme d'une catastrophe naturelle, d'une colère divine, d'une épidémie, d'une invasion étrangère ou d'un soulèvement populaire - est envisagé comme une interruption du cours normal de la vie de la cité, voire comme la pure et simple disparition de la politique au profit de la violence. On connaît la fascination des idéologues fascistes pour l'« ordre nouveau ». On connaît également les réflexions libérales sur la recherche d'un ordre juste. On sait moins, en revanche, que "l'ordre moins le pouvoir " est l'une des formules les plus plébiscitées par les théoriciens de l'anarchisme. Du fascisme à l'anarchisme en passant par le libéralisme, l'ensemble du spectre idéologique communie donc dans la condamnation du désordre.

La désobéissance civile est l'une des figures contemporaines du désordre, puisqu'en transgressant une loi jugée injuste, on porte atteinte à l'ordre public. D'Extinction Rebellyon aux Gilets jaunes en passant par les personnes qui apportent leur aide aux migrants en situation irrégulière, la désobéissance civile opère actuellement une montée en puissance au sein

1. Leo Strauss, Qu'est-ce que la philosophie politique?, Paris, Puf, 2010, p. 18. 
du répertoire contestataire. Ce type d'action illégale et non violente pose à la théorie démocratique un redoutable défi car les désobéissants présentent leur geste comme un signe de vitalité démocratique tandis que ses adversaires y voient une rupture du contrat social. Mais, au-delà de leur opposition politique, partisans et adversaires de la désobéissance civile partagent un même présupposé philosophique : le désordre est mauvais. Pour ses contempteurs, la désobéissance civile risque de précipiter la société dans le chaos généralisé. Les désobéissants répondent qu'ils génèrent un désordre provisoire visant à instaurer un nouvel ordre, plus juste et donc plus stable que l'ancien. In fine les désobéissants sont donc des partisans de l'ordre.

\section{MIEUX VAUT UNE INJUSTICE QU'UN DÉSORDRE}

Le désordre est une crainte ancestrale. La cité idéale de Platon est immuable. Elle ne connaît ni le temps ni le changement, et encore moins la contradiction. La Callipolis est harmonieuse. Elle "ordonne à chacun de remplir sa propre fonction ${ }^{1}$ ". La démocratie ruine la hiérarchie des êtres. Le principe d'égalité sème le désordre en laissant accroire à chacun qu'il vaut autant que les autres. La politique est un ordre qui dérive du savoir du philosophe. La démocratie, règne des opinions et de la délibération, porte atteinte au socle de vérité sur lequel repose la cité idéale. D'où la condamnation du désordre démocratique, qui peut aussi prendre la forme d'un conflit politique que les Grecs désignaient par le terme de stasis. L'Athènes du V viècle avant J.-C. n'était pas la cité calme et civilisée dont Leo Strauss, disciple de Platon, est nostalgique, mais une cité soumise à des divisions, à des affrontements entre le camp aristocratique (les eupatrides) et ceux que l'aristocratie qualifie de kakoü (les "mauvais »). Or, comme l'a montré Nicole Loraux, la cité démocratique cherchait à maintenir cette division ${ }^{2}$. Platon, y voyant une menace de guerre civile, condamnait au contraire le conflit, source de désordre. Platon a fixé, pour longtemps, les termes du débat philosophique. La démocratie est une chose indésirable. Elle sera éventuellement tolérée à condition de la transposer du côté de l'ordre.

1. Platon, La République, Paris, Flammarion, coll. «GF », 1966, p. 185.

2. Nicole Loraux, La Cité divisée. L'oubli dans la mémoire d'Athènes, Paris, Payot, 2005. 
Au XVIII siècle, le philosophe irlandais George Berkeley faisait remarquer que, s'il existe sans conteste des lois qui enfreignent le droit naturel, les individus doivent néanmoins se soumettre à ces lois injustes car ils sont incapables de prévoir les conséquences de leur acte ${ }^{1}$. Bien intentionnée, la désobéissance risque fort de conduire à un résultat encore pire que la situation de départ. Berkeley, comme beaucoup d'auteurs médiévaux, craint davantage la sédition que la tyrannie, car la tyrannie porte atteinte au bien de la communauté tandis que la sédition entraine la dissolution pure et simple de la communauté. Dès le XIII ${ }^{\mathrm{e}}$ siècle, Thomas d'Aquin prônait le respect des lois injustes afin de conjurer la "menace de désordre ${ }^{2} "$.

Ces condamnations du désordre ne doivent pas étonner. Elles furent formulées dans le contexte d'une "société d'ordres " qui voulait que chacun reste à sa place. La hiérarchie de dignité et d'honneur avait un fondement naturel. Gare à celui qui briserait cette belle harmonie.

En reconnaissant la liberté inaliénable de l'individu, en consacrant l'universalité des droits humains et en faisant procéder la souveraineté du peuple, la modernité fournit plusieurs points d'appui à la contestation de l'ordre établi. La révolution démocratique aurait pu produire une forme de réhabilitation du désordre. Il n'en fut rien.

Vers la moitié du XIXe siècle, la culture politique dominante est celle du contrat social. En théorie, le contractualisme peut justifier la désobéissance : lorsque le souverain méconnaît le contrat, le peuple peut résister pour faire valoir ses droits. Mais Thomas Hobbes et Jean-Jacques Rousseau ont opéré un méticuleux travail conceptuel visant à faire disparaitre de la notion de contrat ce qui aurait pu légitimer un droit de résistance et accorder ainsi au désordre une forme de validité démocratique ${ }^{3}$. Rousseau nie l'utilité de la désobéissance tandis que Hobbes en souligne la dangerosité. Pour que la question de la désobéissance légitime puisse être posée, il faut qu'il existe des lois oppressives. Mais Rousseau, en élaborant un système politique parfait où aucune loi ne peut être mauvaise, évacue de facto la possibilité de résister. La loi est l'expression de la volonté générale, or " la volonté générale est toujours droite ${ }^{4}$ ». Dit autrement, la loi est un acte de volonté du peuple. Donc «il ne faut plus demander [...] si la loi

1. George Berkeley, De l'obéissance passive (1712), Paris, Vrin, 1983, paragraphe 9.

2. Thomas d'Aquin, Somme théologique, Ia IIae, qu.96, art. 4.

3. Éric Desmons, Droit et devoir de résistance en droit interne, Paris, LGDJ, 1999, p. 44.

4. Jean-Jacques Rousseau, Du contrat social, Paris, Flammarion, coll. " GF ", 2001, livre II, chapitre 2, p. 68.
Le désordre, essence ou tombeau de la démocratie? Philosopher au prix du doute Manuel Cervera-Marzal 
peut être injuste, puisque nul n'est injuste envers lui-même ${ }^{1}$ ". Rousseau écrit aussi que " la loi est antérieure à la justice, et non la justice à la loi ${ }^{2}$ ", de sorte que la notion de " loi injuste " est d'emblée écartée. Il s'ensuit que la désobéissance aux lois n'a plus de raison d'être.

Hobbes règle le problème de façon tout aussi péremptoire, en affirmant que les lois positives sont toujours justes. À l'état de nature, tous les individus sont égaux. Mais chacun se croit intellectuellement supérieur aux autres et pense donc être le seul habilité à dire ce qui est juste. Tout le monde s'oppose alors sur le sens du juste et de l'injuste, du bien et du $\mathrm{mal}^{3}$. Le désaccord étant total, chacun cherche à imposer par la force son interprétation du juste. Il en résulte un état de guerre de tous contre tous. Pour faire cesser cette guerre, Hobbes postule la nécessité d'un souverain absolu, Léviathan, qui met fin au désaccord généralisé en tranchant les débats. "La loi est l'expression de la volonté du souverain en ce qui concerne le juste et l'injuste ${ }^{4}$. » Par définition, toute loi posée par le souverain est donc juste. Et la désobéissance, comme chez Rousseau, n'a aucune raison d'être. On notera cependant avec Jacques Rancière que, par un ironique retournement de l'histoire, les théories du contrat social, qui avaient été élaborées " pour fonder et protéger les droits des princes face aux révoltes aristocratiques, populaires ou religieuses, ont servi à construire la mise en scène révolutionnaire des droits du peuple ${ }^{5}$ ».

La formule de Goethe est connue : "J'aime mieux une injustice qu'un désordre. " Autrement dit, la condamnation d'un innocent serait préférable à la mise en question des autorités et, par conséquent, quand bien même on reconnaîtrait qu'une loi est inique, il faudrait s'y soumettre. Le présupposé étant que le désordre permettrait la réalisation d'une plus grande injustice ${ }^{6}$. C'est l'interprétation classique de cette formule, et c'est le sens qu'elle a chez les penseurs conservateurs qui, à l'instar de Maurice Barrès, accusaient les dreyfusards de semer la pagaille en jetant le soupçon sur l'armée. Il faut cependant innocenter Goethe de l'accusation de cynisme qu'on adresse à cette philosophie. La phrase doit être replacée dans son contexte. Goethe a en réalité écrit : "Cela tient finalement à ma

1. Ibid., livre II, chapitre 6, p. 77.

2. Jean-Jacques Rousseau, Euvres complètes, Paris, Gallimard, 1959, livre III, p. 329.

3. Thomas Hobbes, Léviathan, Paris, Sirey, 1971, chapitre 18.

4. Ibid., chapitre 26.

5. Jacques Rancière, Et tant pis pour les gens fatigués, Paris, Éditions Amsterdam, 2009, p. 181. 2012.

6. Robert Legros, "Goethe et le siège de Mayence ", in Philosophie Magazine, 24 septembre 
nature, je préfere commettre une injustice que de tolérer un désordre. » Tandis qu'à la fin du siège de Mayence, en 1793, les Français avaient obtenu le droit de quitter la ville sans être inquiétés, la foule tenta de lyncher un soldat français qu'elle accusait, à tort, d'avoir incendié le doyenné de la cathédrale. Goethe s'interposa et évita à ce malheureux une mort assurée. Interrogé plus tard sur les motifs de son intervention, il répondit que mieux valait une injustice qu'un désordre. Entendons par là qu'il préférait laisser s'échapper un coupable potentiel (une injustice) que d'accepter qu'il soit livré à une foule en furie (un désordre). Tout suspect est présumé innocent et a droit à un procès équitable. Rien à voir, donc, avec l'acception conservatrice de cette formule. Mais laissons Goethe de côté et tenons-nous en à l'acception courante du " mieux vaut une injustice qu'un désordre ".

Cette sentence pose trois problèmes. D'abord, il est dérangeant que les défenseurs de l'injustice soient rarement ceux qui ont à la subir. Le philosophe Jean-Marie Muller dénonce à ce titre les "nombreux libéraux, qui sont prêts à dénoncer l'injustice et à la combattre par les moyens constitutionnels, mais qui n'accepteront pas de franchir le seuil qui les ferait entrer dans l'illégalité. Pour eux, l'ordre est indivisible et toute désobéissance ne peut être qu'un facteur de désordre. [...] L'homme libéral, en effet, souhaite que les opprimés puissent obtenir la reconnaissance de leurs droits, mais il possède une patience et un optimisme qui lui permettent d'attendre et de faire confiance aux pouvoirs établis. Aussi juge-t-il les méthodes d'action directe, et notamment les actions de désobéissance civile, comme des actes extrémistes et peu raisonnables ${ }^{1}$ ".

Deuxièmement, la sentence goethienne suppose qu'injustice et désordre entretiennent un rapport d'exclusion, ce qui est parfois - mais pas systématiquement ! - le cas. Souvent, injustice et désordre vont de pair - par exemple lorsque des policiers dispersent brutalement une manifestation pacifique -, de sorte que l'alternative formulée par Goethe n'a aucune raison d'être. Platon considère d'ailleurs que désordre et injustice sont synonymes. Enfin, Goethe reste prisonnier du préjugé péjoratif à l'encontre du désordre. Gandhi, pourtant inlassable pourfendeur des injustices, ennemi numéro un de la domination britannique, reste lui aussi un défenseur de l'ordre.

Les adversaires politiques du leader nationaliste reprochèrent à la noncoopération d'exciter les masses et d'entraîner des violences. Il est vrai que,

1. Jean-Marie Muller, Stratégie de l'action non violente, Paris, Seuil, 1981, p. 77. 
malgré l'affichage d'intentions non violentes, ses campagnes d'agitation publique conduisirent à plusieurs reprises à des émeutes sanglantes. En février 1919, Gandhi perdit le contrôle du mouvement qu'il avait initié contre les lois Rowlatt qui restreignaient considérablement les libertés individuelles. Il s'ensuivit des émeutes, des pillages et des vols à travers toute l'Inde. La non-coopération tourna à nouveau à l'émeute en 1921. Ses adversaires firent valoir que, si Gandhi n'avait pas anticipé ces répercussions violentes, alors il manquait de prévoyance. $\mathrm{Si}$, au contraire, il s'y attendait, alors c'est qu'il était prêt à accepter les conséquences violentes de l'action non violente.

Gandhi rétorque que ce n'est pas la désobéissance civile qui constituait ici un danger pour l'ordre démocratique. En effet, la désobéissance ne fit que révéler la violence, latente, qui existait déjà au sein de la société coloniale. Mais elle n'en est pas la cause à proprement parler, puisqu'elle ne fait que ressortir ce qui était déjà présent, de manière sous-jacente : "Le fait que la désobéissance civile se transforme en désobéissance violente n'est pas, je dois l'avouer à regret, une situation improbable. Mais je sais qu'elle n'en sera pas la cause. La violence est déjà là qui corrode tout le monde politique. La désobéissance civile ne sera qu'un processus de purification et fera peut-être remonter à la surface ce qui est enfoui dans ce monde ${ }^{1}$. " Les injustices antérieures à la campagne de satyagraha furent la cause réelle des émeutes. Gandhi affirme ainsi que " la désobéissance civile ne conduit jamais à l'anarchie ${ }^{2}$ ". À ses yeux, le désordre suscité par la désobéissance est limité, ponctuel, provisoire et, surtout, il vise l'instauration d'un nouvel ordre, plus humain et donc plus durable que l'ordre colonial. À tous égards, Gandhi conserve un préjugé négatif à l'égard du désordre. Il s'excuse pour les troubles commis. Une autre optique est pourtant possible, comme y invite le philosophe Claude Lefort.

\section{ACCUEILLIR L'INDÉTERMINATION POLITIQUE}

Claude Lefort, à l'instar de Hannah Arendt, considère que les événements du $\mathrm{XX}^{\mathrm{e}}$ siècle, en particulier l'avènement des régimes totalitaires, font vaciller la conception dominante de la politique. "Ils créent à la pensée l'exigence d'un nouveau départ ${ }^{3}$. » Lefort est l'auteur qui est allé

1. Gandhi, Résistance non violente, Paris, Buchet-Chastel, 2007, p. 346.

2. Ibid., p. 269.

3. Claude Lefort, Essais sur le politique, Paris, Seuil, 2001, p. 13. 
le plus loin dans la tentative de nouer le désordre et la démocratie sous un jour positif (Platon aussi assimilait les deux, mais pour condamner le désordre démocratique). Une chose est d'admettre qu'il faut quelques désordres (révolution contre le roi, insurrection contre le dictateur, résistance à l'invasion étrangère) pour instaurer un ordre démocratique. La plupart des philosophes admettent cette vérité banale : le désordre précède la démocratie. Autre chose est d'affirmer, comme ose le faire Lefort, que le désordre est constitutifà la démocratie. Car une telle affirmation revient à briser le nœud gordien entre ordre et démocratie. Cette dernière n'est plus uniquement un régime politique ; elle est aussi le chaos qui vient troubler, perturber, secouer et déstabiliser les institutions. Dans un texte rédigé à l'occasion du vingtième anniversaire de Mai 68, Claude Lefort écrit ainsi :

L'image du désordre suscite le plus souvent réprobation ou indignation. Comme on le sait, elle fut habilement exploitée par l'extrême droite et le fascisme, qui condamnaient sans relâche la licence, l'inconsistance, la turbulence, les déchirements de la "société bourgeoise " et s'étaient faits les champions d'un ordre nouveau. Je m'emparai donc de cette image pour montrer que le mouvement de Mai ne s'attaquait pas à l'ordre établi pour lui substituer un ordre meilleur - lequel ne s'impose que par la terreur et une idéologie de fer - mais qu'il revendiquait un désordre à l'intérieur de la société, la permanence d'une contestation des pouvoirs en place ${ }^{1}$.

Il faut ici saluer l'audace de Claude Lefort. Il faut aussi faire un peu d'exégèse, car Lefort exhume à propos de Mai 68 une thèse qu'il avait déjà esquissée en commentant le conflit ayant opposé la plèbe romaine aux patriciens. Dans Machiavel. Le travail de l'ouvre, thèse de doctorat d'État soutenue en 1971 après dix années de travail, Lefort entreprend une véritable réhabilitation démocratique du désordre. Au neuvième chapitre du Prince, après avoir rappelé que tout nouveau prince parvient au pouvoir en prenant appui soit sur le peuple soit sur les aristocrates, le Florentin écrit qu'en " toute cité on trouve ces deux humeurs différentes, desquelles la source est que le peuple désire ne pas être commandé ni opprimé par les Grands et les Grands désirent commander et opprimer le peuple ; et de ces deux différents appétits naît dans les cités un de ces trois effets : ou principauté, ou liberté, ou licence ${ }^{2} »$. Ce conflit n'est pas circonstanciel. Il ne saurait être dépassé dans l'utopie d'une société harmonieuse, comme

1. Claude Lefort, "Relecture ", in La Brèche, suivi de Vingt ans après, Paris, Fayard, 2008, p. 285.

2. Nicolas Machiavel, Le Prince, Sainte-Foy, Le Griffon d'Argile, 1984, p. 39. 
l'ont cru à tort les marxistes et les saint-simoniens, qui espéraient remplacer le gouvernement des hommes par l'administration des choses. Car, ainsi que l'explique Lefort :

C'est bien d'une opposition constitutive du politique qu'il faut parler, et irréductible à première vue, car ce qui fait que les Grands sont les Grands et que le peuple est le peuple ce n'est pas leur fortune, pas leurs mœurs, ou un statut distinct associé à des intérêts spécifiques et divergents; c'est, Machiavel le dit sans ambages, que les uns désirent commander et opprimer et les autres ne l'être pas. Leur existence ne se détermine que dans cette relation essentielle, dans le heurt de deux "appétits " par principe également "insatiables " ${ }^{1}$.

Après avoir exhumé l'idée (machiavélienne) que la liberté était le fruit des tumultes du peuple, Lefort prolonge Machiavel en ajoutant, contre les révolutionnaires de papier, qu'on ne peut plus "se contenter de dire qu'il y a dans le désordre de quoi fonder un nouvel ordre ${ }^{2} »$. Il faut aller plus loin, retourner le stigmate et reconnaître le caractère permanent du désordre. Car ceux qui tentent "d'éliminer le désordre " causent toujours une "dégradation de la loi et de la liberté " ${ }^{3}$. Lefort ajoute que "le désordre, au sens vrai du terme, n'est pas la pure discorde, le tumulte où s'entrechoquent les intérêts particuliers, car ce désordre-là s'accommode fort bien, comme il en va à Florence, de l'apparence de l'ordre, c'est-à-dire de l'équilibre acquis des forces sociales ; il est l'opération du désir qui tient ouverte la question de l'unité de l'État, et, en la dévoilant, contraint ceux qui le dirigent à remettre en jeu son destin ${ }^{4}$ ». Ou comme le dit Miguel Abensour, disciple de Claude Lefort : «La vérité de la démocratie se trouve dans son insurrection contre l'État qui, lui, cherche à neutraliser ou à domestiquer les désordres démocratiques ${ }^{5}$."

Seuls les désordres démocratiques semblent d'ailleurs en mesure d'ouvrir une faille où les hommes puissent s'engouffrer pour tisser de nouvelles relations et abolir l'ancien ordonnancement hiérarchique du social. Ainsi par exemple en 1871, lors du siège de la capitale par les troupes versaillaises, la Commune de Paris donne au peuple l'occasion de se nouer de façon inédite à sa ville. Aux boulevards haussmanniens dont l'imposante largeur a été souhaitée par les puissants pour facilement disperser les ras-

1. Claude Lefort, Machiavel. Le travail de l'ouvre, Paris, Gallimard, 1972, p. 382.

2. Ibid., p. 478 .

3. Ibid.

4. Ibid.

5. Miguel Abensour, "Démocratie insurgeante et institution ", in Martin Breaugh, Francis Dupuis-Déri (dir.), La Démocratie au-delà du libéralisme, Québec, Athéna Éditions, 2009, p. 185. 
semblements populaires à coups de canons, les communards répondent par une réappropriation de l'espace urbain. Les rassemblements collectifs, les fêtes, les concerts et les réunions se multiplient, les femmes intègrent la scène politique, bref, le quotidien sort de la sphère privée et le peuple bouleverse le partage du sensible. Le siège de la capitale désintègre les rôles traditionnels. Les places autrefois assignées à chacun en fonction de son sexe, son statut, sa classe et sa race disparaissent. L'insurrection révolutionnaire produit une resocialisation politique. L'expérience communale, brève mais intense, révèle combien la politique est guidée par la recherche d'un lien non hiérarchique plutôt que par la mise en œuvre d'un ordre coercitif.

La philosophie lefortienne, mieux que toute autre, rend intelligibles les épisodes révolutionnaires. Cette faculté est liée à un second déplacement conceptuel, qui conduit Claude Lefort à envisager la démocratie en tant que "formes de société " et donc à ne pas la réduire aux "institutions politiques " que se donne une telle société, et qui en constituent le fondement mais non la totalité. La démocratie ne se limite pas à un régime. Elle est une totalité articulée qui comporte aussi des formes de vie, des expérimentations sociales et, de manière encore plus fondamentale, une dimension symbolique. Car la mise en forme de l'espace social "est en même temps une mise en sens et une mise en scène. Mise en sens car l'espace social se déploie comme espace d'intelligibilité, s'articulant suivant un mode singulier de discrimination du réel et de l'imaginaire, du vrai et du faux, du juste et de l'injuste, du licite et de l'interdit, du normal et du pathologique. Mise en scène, car cet espace contient une quasi-représentation de lui-même dans sa constitution aristocratique, monarchique ou despotique, démocratique ou totalitaire ${ }^{1}$ ». Cet élargissement du regard posé sur la démocratie rend possible une réhabilitation du désordre.

Mais cette réhabilitation souffre cependant d'une confusion théorique et d'une aporie pratique. Sur le plan de la théorie, Claude Lefort ne cesse de confondre désordre et conflit. Quand il parle de la plèbe romaine comme quand il parle des soixante-huitards, il utilise indistinctement ces deux notions. Jamais il ne problématise les rapports entre désordre et conflit, de sorte que son lecteur a le sentiment qu'il s'agit d'une identité conceptuelle. Pourtant, le conflit va parfois de pair avec l'ordre plutôt qu'avec le désordre. La lutte des classes a structuré les sociétés modernes. Autrement dit, le conflit entre capital et travail a mis de l'ordre dans les pays industrialisés. Certes, à l'occasion d'une grève durement réprimée ou
Le désordre, essence ou tombeau de la démocratie? Philosopher au prix du doute Manuel Cervera-Marzal

1. Claude Lefort, Essais sur le politique, Paris, Seuil, 1986, p. 20. 
de la séquestration d'un patron, l'affrontement entre la bourgeoisie et le prolétariat a pu être source de désordre. Mais, dans l'ensemble, en distinguant deux pôles et en fixant à chacun un rôle clairement défini, le conflit a ordonné le monde social. De la même façon, le conflit entre l'Est et l'Ouest a conféré au monde de la guerre froide une forme de stabilité, de lisibilité, bref, un ordre. La confusion entre désordre et conflit est gênante, car si Lefort s'intéresse au conflit démocratique, alors il se contente de mettre ses pas dans ceux de La Boétie, Machiavel, Marx, et de cheminer avec Miguel Abensour, Giorgio Agamben, Étienne Balibar, Ernesto Laclau, Chantal Mouffe et Jacques Rancière. Son originalité est donc limitée, puisqu'il existe une vaste constellation de penseurs soucieux de la dimension agonistique de la démocratie. En revanche, si Lefort s'intéresse au désordre démocratique, il ouvre une voie originale.

La seconde difficulté à laquelle se heurte Claude Lefort est d'ordre pratique. Autant sa théorie du désordre est séduisante au plan conceptuel, autant on voit difficilement à quelle application elle peut donner lieu. Sa politique du désordre semble en effet impossible à concrétiser. Elle est un aveu d'impuissance. Expliquons pourquoi. Dans l'article déjà cité sur Mai 68, Lefort définit très exactement le désordre comme « la permanence d'une contestation des pouvoirs en place ». L'élément central réside ici dans l'adjectif " permanent ". Lefort pense la politique contre les pouvoirs, c'est-à-dire contre toute forme d'organisation qui tendrait à conférer à ses membres une forme de pouvoir. Pour mieux comprendre la position de Lefort, il convient de revenir sur la querelle qui l'opposa à Cornelius Castoriadis quant au problème de l'organisation. Une divergence de fond, récurrente au sein de Socialisme ou Barbarie, portait sur la question du pouvoir et la pertinence de la mise en place d'une organisation militante. Castoriadis, soucieux d'efficacité, défendait la nécessité d'un parti. Lefort, à l'inverse, était tellement inquiet du risque de bureaucratisation qu'il refusait l'établissement du moindre organisme centralisateur. La thèse de Lefort conduit à mettre en cause l'idée même d'organisation, suspectée de pervertir la démocratie. L'aporie lefortienne apparaît dès qu'on se pose la question concrète de l'action collective. En effet, comment militer s'il n'est plus possible de s'investir dans une organisation? Comment modifier la réalité si on refuse d'occuper la moindre position de pouvoir ? Cornelius Castoriadis a pointé la difficulté que pose la thèse lefortienne : bien que légitime, sa mise en garde contre le risque bureaucratique semble conduire à l'inaction. Une politique du désordre, guidée par la méfiance permanente 
envers toute organisation, conduit nolens volens au refus de l'action et donc au refus de la politique.

Au terme de ce parcours, on s'aperçoit que la démocratie ne peut être pensée sur le mode exclusif de l'ordre ni sur le mode exclusif du désordre. L'économie politique de la démocratie se tient ainsi dans l'entrelacs de la stabilité et de la nouveauté, de la conservation et de la transformation, du temps long et du temps court, de la durée et de l'événement, du pouvoir et de sa contestation. Ordre et désordre font l'objet d'une mystérieuse imbrication sur laquelle s'érige la démocratie. En conséquence, cette dernière n'est pas identifiable à un assemblage institutionnel définitivement figé. Elle n'existe qu'en se renouvelant. Cependant, la démocratie n'est pas non plus une frénésie destructrice qui abolirait immédiatement tout germe d'institutionnalisation. Elle se donne provisoirement des institutions, car elle ne peut exister qu'avec un minimum de stabilité. La démocratie est donc prisonnière d'une tension féconde entre sa dimension ordonnée et sa dimension désordonnée.

Le caractère insurmontable de cette tension fait de la démocratie le régime de l'indétermination. La démocratie est une aventure qui accueille l'incertitude et qui s'expose au risque. La démocratie est le nom d'un projet, disait Castoriadis. Quant à Lefort, il voyait en elle une forme de société où "le pouvoir, la loi, la connaissance se trouvent mis à l'épreuve d'une indétermination radicale, société devenue théâtre d'une aventure immaitrisable, telle que ce qui se voit institué n'est jamais établi, le connu reste miné par l'inconnu, le présent s'avère innommable ${ }^{1}$ ». 